\title{
Les primitifs sémantiques dans la langue. Leur place et leur fonction
}

\author{
Arkadiusz Koselak \\ Université Paul Verlaine de Metz \\ a.koselak@laposte.net
}

La recherche de primitifs conceptuels préoccupe depuis longtemps les chercheurs du champ des sciences humaines. En linguistique, c'est Anna Wierzbicka, une linguiste australienne d'origine polonaise, qui a mis en place une méthodologie et un cadre théorique structurés pour mettre en évidence les primitifs sémantiques. Les travaux de Wierzbicka et de ses collègues ont permis l'élaboration d'un outil linguistique impressionnant : la métalangue sémantique naturelle.

Les primitifs sémantiques [semantic primes], parce qu'ils sont primitifs, doivent jouer un rôle important dans la structure sémantique des langues, autrement dit, dans la structure des images linguistiques du monde. La nature même des primitifs, c'est-à-dire leur caractère primaire et l'impossibilité de les définir, présuppose que les primitifs participent à la catégorisation linguistique. L'importance de leur rôle est démontrée par le principe même de leur recherche : lorsqu'un primitif est postulé, on teste sa pertinence de manière lexicologique et on vérifie s'il est vraiment impossible de le réduire sémantiquement à l'aide des concepts au moins aussi simples que lui-même.

Lorsque l'on observe la métalangue sémantique naturelle et les primitifs sémantiques qui la composent, on se rend rapidement compte que les primitifs ne sont pas tous de la même nature. Certains sont peu saturés sémantiquement ${ }^{1}$ comme par exemple QUELQUE CHOSE ou FAIRE ${ }^{2}$, d'autres sont beaucoup plus saturés comme par exemple CORPS. Enfin, d'autres renvoient à des relations très spécifiques entre les concepts dans la construction de l'image du monde comme par exemple AU-DESSUS. Autrement dit, certains primitifs sont catégorisateurs et d'autres non, ils jouent donc un rôle différent dans l'organisation sémantique. L'objet de ce travail est d'examiner les primitifs sémantiques de la métalangue sémantique naturelle du point de vue du rôle qu'ils jouent dans la structuration de l'image linguistique du monde. Il ne s'agit pas de présenter une recherche achevée mais plutôt de poser des jalons de recherches plus globales sur les primitifs. La problématique abordée ici doit permettre d'une part, de mieux comprendre la nature des primitifs sémantiques par leurs implications dans la structuration sémantique et, d'autre part, comprendre mieux cette structuration. On considère que les primitifs sont extraits des langues naturelles, mais on s'est en effet peu préoccupé des primitifs dans leurs langues d'origine. Pour dire les choses autrement, il semble nécessaire d'étudier les primitifs également en dehors de la métalangue sémantique naturelle et des explicitations, de les envisager en tant que les éléments de la langue. Nous verrons qu'aborder les primitifs du point de vue de leur rôle structurant soulève des questions plus générales sur les recherches portant sur la métalangue sémantique naturelle.

Les analyses présentées ici concernent la version française de la métalangue sémantique naturelle mais, a priori, certains résultats peuvent être généralisables aux MSN dérivées d'autres langues.

La présentation est articulée en trois parties. Dans un premier temps, j'aborderai les notions générales mobilisées par la recherche avec notamment les fondements de la théorie de primitifs sémantiques, la catégorisation et l'image linguistique du monde. Dans un deuxième temps, j'examinerai les primitifs sémantiques du point de vue de leur fonction. Et enfin, dans un troisième temps, $j$ 'interrogerai, de manière générale, les implications de la recherche présentée dans la théorie de la métalangue sémantique naturelle. 


\section{Les fondements théoriques : les primitifs, les catégories et l'image linguistique du monde}

Il n'est pas possible de présenter ici la théorie complète de la métalangue sémantique naturelle ${ }^{3}$, je vais en rappeler les grands principes dont j'aurai besoin pour développer mon propos. La problématique des primitifs sémantiques, développée en linguistique de manière systématique par Anna Wierzbicka, a une tradition philosophique ancienne qui remonte au moins jusqu'à Leibniz et Descartes (Wierzbicka, 1972). On peut mettre en rapport les postulats leibniziens avec les postulats cognitivistes contemporains. En effet, Leibniz postulait l'existence des « atomes » de la pensée, c'est-à-dire des idées simples, universelles qui serviraient à la construction de toutes les autres idées complexes alors que les approches cognitivistes contemporaines ${ }^{4}$ postulent une universalité des mécanismes soutenant la cognitition. Mais le parallèle est encore plus précis dans la mesure où Leibniz, en postulant le caractère inné et l'universalité humaine de certains contenus de la pensée, évoquait nécessairement les mécanismes de la création de la pensée complexe.

L'introduction de la problématique des primitifs conceptuels à la linguistique nécessite une adaptation évidente : la présence matérialisée des idées simples postulées dans la langue est requise. Wierzbicka a en effet postulé que pour que l'on puisse parler des primitifs sémantiques, il faut qu'ils soient présents, de manière formelle, dans la langue, peu importe la nature catégorielle de cette présence ${ }^{5}$. Ce postulat a été par la suite formalisé dans le principe de lexicalisation forte (Goddard, 1994). Les primitifs sémantiques expriment donc des idées simples sur la surface de la langue. Ils sont primitifs parce qu'ils ne sont pas décomposables à l'aide des unités au moins aussi simples qu'eux, selon le principe définitoire mis en évidence déjà par Aristote. Ce principe est important dans la mesure où l'on risque la confusion entre deux points importants concernant les primitifs sémantiques. On peut percevoir, intuitivement un lien entre certains primitifs, comme par exemple VOULOIR et BON ou encore QUELQU'UN et JE. Mais ce qu'il y a de commun entre eux n'est pas matérialisé dans la langue, autrement dit la composante commune n'est pas sémantique mais purement conceptuelle. Cela n'empêche pas que l'on puisse approcher, à l'aide des unités linguistiques complexes, le contenu sémantique des primitifs. Nous verrons par la suite que cela serait très utile. Dans la mesure où les primitifs sémantiques expriment des idées innées, on s'attend à ce qu'ils soient universels, c'est-à-dire présents dans toutes les langues humaines. Bien évidemment ce postulat n'est pas vérifiable en tant que tel, cependant les études menées sur les langues appartenant à des familles linguistiques diverses ${ }^{6}$ confirment cette hypothèse et il n'y pas de raisons de penser qu'il n'en soit pas ainsi. Il est nécessaire de préciser que ce n'est pas parce qu'une unité linguistique possède des équivalents dans toutes les langues du monde qu'elle exprime un sens primitif. Ainsi par exemple le concept de mère ou de vent semble être présent dans toutes les langues, mais ces deux concepts sont bien décomposables à l'aide des primitifs (Wierzbicka, 1989).

Les primitifs sémantiques possèdent leur propre combinatoire, elle aussi supposée primitive. Comme cette combinatoire ne concerne pas la problématique abordée, il n'en sera pas question ici. ${ }^{7}$

La métalangue sémantique est considérée comme naturelle d'une part par opposition aux métalangages abstraits et d'autre part parce qu'elle dérive directement des langues naturelles. Les primitifs sémantiques présentent toutefois une différence majeure avec des unités de la langue : la polysémie omniprésente dans les langues naturelles est ici supprimée et on ne reconnait qu'une valeur à chaque primitif. Cette valeur ne pouvant pas être définie, on l'indique à l'aide des structures qui sélectionnent la valeur souhaitée. Ainsi, par exemple, on indiquera la valeur retenue du primitif ARRIVER à l'aide de la structure « quelque chose est arrivé à $X »$. Nous verrons que cette procédure ne désambigüise pas toujours suffisamment les primitifs. On reproche fréquemment à la métalangue naturelle de ne pas rendre compte de la polysémie qui est un phénomène omniprésent dans la langue, mais de telles critiques confondent deux niveaux d'analyse différents. La métalangue sémantique naturelle est tout d'abord un outil et les études sur les primitifs sont des analyses d'un outil et de ses possibilités d'application avec, bien évidemment, les postulats théoriques qui les sous-tendent. La suppression de la polysémie est empiriquement nécessaire dans l'outil lui-même, si cette suppression n'avait pas été effectuée, la métalangue sémantique naturelle n'aurait pas de sens en tant qu'outil. Par contre, cette réduction au niveau de l'outil ne préjuge en rien sur des capacités de la métalangue sémantique naturelle à rendre compte de la polysémie dans la langue. 
Dans la mesure où mes réflexions portent sur les primitifs en tant qu'outils, je me concentrerai sur les valeurs sémantiques portées par les primitifs, je garderai donc la suppression de la polysémie.

Les études sur les métalangues sémantiques naturelles sont toujours en cours, aucune version n'est à considérer comme définitive (Wierzbicka, 1996), on peut cependant penser que les dernières versions s'approchent du définitif. La dernière version «officielle» de la MSN française est celle de Peeters (2006) et se présente ainsi :

Substantifs : JE, TU, QUELQU'UN, QUELQUE CHOSE, GENS, CORPS

Déterminants : CE, MEME, AUTRE

Quantificateurs : UN, DEUX, IL Y A ... QUI, BEAUCOUP, TOUT

Prédicats mentaux : SAVOIR/CONNAITRE, PENSER, VOULOIR, (SE) SENTIR, ENTENDRE, VOIR

Actions, évènements, mouvement : FAIRE, ARRIVER, BOUGER

Discours : DIRE, MOTS, VRAI

Existence et possession : IL Y A, AVOIR,

Vie et mort : VIVRE, MOURIR

Evaluateurs : BIEN, MAL

Descripteurs : GRAND, PETIT

Espace : OU (ENDROIT), ICI, AU-DESSUS, EN-DESSOUS, LOIN, PRES, COTE, DANS (DEDANS), TOUCHER (SE TOUCHER)

Temps : QUAND (MOMENT, FOIS), MAINTENANT, AVANT, APRES, LONGTEMPS, PEU DE TEMPS, POUR UN TEMPS, EN UN MOMENT,

Concepts logiques : NE...PAS, POUVOIR, PEUT-ETRE, A CAUSE DE (PARCE QUE), SI

Intensifieur, augmentateur : TRES, PLUS

Similarité : COMME

Taxonomie, partonomie : TYPE DE, PARTIE (AVOIR DES PARTIES)

La métalangue sémantique naturelle n'est pas une simple liste, les primitifs s'organisent en catégories. Certaines catégories des primitifs se couvrent avec les domaines sémantiques, comme nous le verrons par la suite.

Avant de clore cette présentation rapide des primitifs sémantiques, il est encore nécessaire d'introduire une notion théorique particulière concernant les primitifs sémantiques, il s'agit d'allolexie. L'alollexie désigne le phénomène de variation morphologique des primitifs imposée par les contextes. Deux types d'allolexie sont reconnus (Goddard, 2002): l'allolexie positionnelle et l'allolexie combinatoire. L'allolexie positionnelle peut être illustrée par l'alternance de pronoms je et me pour exprimer le primitif JE. Tous les deux ont le même sens, la forme doit changer selon la position pour que les formules obtenues puissent être jugées correctes dans la langue dont la MSN est dérivée (principe d'isomorphie). Le mécanisme de l'allolexie combinatoire se fonde sur des compatibilités de concaténation et le principe d'isomorphie indiqué supra. Ainsi par exemple la concaténation ce quelque chose est peu naturelle en français et l'on peut la remplacer par la formule plus naturelle cette chose sans que l'on doive considérer qu'il y ait un changement de sens.

Il convient également de préciser ce que l'on comprendra ici par la catégorisation. Le présent travail s'inscrit dans une perspective cognitiviste en général sans toutefois référer à des travaux précis. On comprendra ici la catégorisation au sens quelque peu naïf, telle qu'elle est mobilisée par des procédés définitoires et explicitants. La forme la plus simple, la plus naïve d'expliquer, définir ou expliciter est justement la catégorisation. Ainsi par exemple, on considérera que le primitif CHOSE est un catégorisateur parce que, a priori, toutes les choses, peuvent être expliquées par une formule « $\mathrm{X}$ est un genre de chose ». CHOSE subsume donc tous les objets du monde conceptualisés linguistiquement en tant que tels. J'utilise le terme "catégorisateur », ce qui permet d'une part d'éviter des termes comme hypéronyme ou archilexème provenant d'autres approches linguistiques et, d'autre part, de démarquer les structures et les relations sémantiques que je tente de mettre en évidence des relations envisagées comme lexicales. La notion de catégorisation mobilise nécessairement la notion de domaines conceptuels. On comprendra ici par le domaine, une portion de l'image linguistique du monde constituée des unités conceptuelles ayant 
en commun une composante essentielle de leur nature sémantique. Bien évidemment il n'est pas possible de déterminer précisément ce qui est compris par 'essentiel', mais il suffira ici de dire, que sans cette composante les unités envisagées ne seraient pas ce qu'elles sont. La proximité sémantique implique que tous les éléments d'un domaine conceptuel peuvent, a priori, être expliqués (ou explicités) à l'aide d'un même schéma définitoire, ce schéma reflétant lui-même la structure du domaine correspondant (Wierzbicka, 1985). Les catégorisateurs, dans l'approche cognitiviste qui est adaptée dans ce travail, organisent des concepts au sens des objets de l'image linguistique du monde. La notion de l'image linguistique du monde qui est adaptée ici est empruntée à l'école ethnolinguistique de Lublin et se comprend comme interprétation (conceptualisation) linguistique de l'entour (Maćkiewicz, 1999). Cette image s'exprime dans la structure sémantique.

\section{Les primitifs et la structure sémantique}

L'observation des primitifs sémantiques permet de les regrouper en trois ensembles: les primitifs «catégorisateurs», les primitifs «non-catégorisateurs» et les primitifs «modalisateurs». Cette tripartition est, assez évidente, mais il est difficile d'y inclure tous les primitifs pour des raisons diverses que nous verrons également plus tard.

\subsection{Les primitifs catégorisateurs}

L'hypothèse de départ était que les primitifs sémantiques jouent un rôle important dans l'organisation sémantique. Nous allons voir maintenant que seulement certains primitifs peuvent être considérés comme des catégorisateurs.

Les deux premiers primitifs ayant clairement un statut de catégorisateurs sont QUELQUE CHOSE et QUELQU'UN. Le statut de ces deux primitifs était évident depuis la première version de la métalangue sémantique naturelle publiée par Wierzbicka $(1972)^{9}$ et ils ne semblent pas poser de problèmes dans aucune des langues étudiées. Ces deux primitifs permettent une première catégorisation : «la distinction entre 'qui' et 'quoi', 'quelqu'un' et 'quelque chose' montre la forme la plus fondamentale de la catégorisation humaine $\gg^{10}$ (Wierzbicka, 1996 : 38). Apparemment, dans toutes les cultures, les objets humains sont conceptualisés différemment que les autres objets du monde ${ }^{11}$. Cette opposition fondamentale se retrouve dans le métalangage abstrait élaboré par R. Jackendoff (1985). L'importance du concept de chose se trouve également soulignée dans la grammaire cognitive de Langacker (2002) pour qui chose est le concept auquel renvoient prototypiquement les prédicats nominaux.

Une telle tentative n'a pas été faite, mais on peut considérer que tous les objets du monde linguistiquement conceptualisés peuvent être définis à l'aide de la formule explicitante commençant par : «c'est un genre de chose... ». Il en va de même pour des objets humains qui peuvent être expliqués par une formule commençant par : «c'est quelqu'un qui... ». Notons que c'est possible parce que QUELQUE CHOSE aussi bien que QUELQU'UN sont très peu saturés sémantiquement.

La catégorisation fondamentale en PERSONNE et CHOSE est universelle et il s'agit bien là d'une interprétation du monde qui diffère des interprétations objectivisées scientifiquement dans lesquelles un humain peut être considéré comme un objet physique du monde au même genre qu'une chose. Mais cette catégorisation universelle n'est pas, bien évidemment, suffisante pour organiser tous les objets de l'image linguistique du monde et c'est notamment la catégorie de CHOSE qui doit être portionnée plus en avant. Mais ces catégorisations seront d'un autre niveau dans la mesure où elles seront spécifiques culturellement. Par exemple, certaines langues ont recours au concept d'animal, alors que d'autres départagent les objets non humains en comestibles ou non-comestibles. En français, comme dans les autres langues européennes, il s'agit d'un problème complexe dans la mesure où il faut encore déterminer si le domaine 'animal' appartient au domaine de CHOSE ou est-ce qu'il se situe au même niveau que CHOSE. Un autre problème qu'on peut relever est celui de concepts abstraits : peut-on les catégoriser aussi comme CHOSE ? 
Il est important d'observer ici, que les primitifs sémantiques ne sont pas suffisants pour rendre compte de la structure sémantique de l'image linguistique du monde du français (ni d'aucune autre langue a priori). Il ne s'agit cependant pas d'une insuffisance à proprement dit, mais du fait que l'on identifie deux niveaux de catégorisation : universelle et culturellement spécifique.

Les primitifs regroupés comme 'prédicats mentaux' ${ }^{12}$ on eux aussi un pouvoir catégorisant, mais il est plus difficile à mettre en évidence que celui de deux primitifs que nous venons de voir, certainement compte tenu de leur caractère immatériel. Les travaux, aussi bien linguistiques que psychologiques, portant sur les concepts affectifs mettent en évidence l'existence d'au moins quatre domaines conceptuels : cognitif, volitionnel, perceptif et physique (Koselak, 2007a). Si l'existence de ces domaines semble indiscutable, il n'est cependant pas aisé de déterminer leurs catégorisateurs.

Commençons par le domaine cognitif. Il semble être catégorisé par le primitif PENSER, mais si c'est le cas, quel rôle remplit le primitif SAVOIR/CONNAITRE ? Peut-être faut-il penser que les deux primitifs catégorisent le domaine cognitif? L'hypothèse séduisante consisterait à dire que la différence entre les deux serait en fait une différence d'agentivité, ce qui nous permettrait de retrouver, dans le domaine cognitif, l'opposition fondamentale actif/passif qui traverserait tous les domaines (Bossong, 1998). SAVOIR serait alors patientif et PENSER agentif ${ }^{13}$. Cette vision du domaine cognitif correspond également aux deux modèles naïfs du fonctionnement mental mis en évidence par Dąbrowska (1994) et elle a été démontrée pour les concepts affectifs (Koselak, 2007a). L'hypothèse qui vient d'être énoncée demande à être vérifiée par une étude systématique des prédicats qui conceptualisent les activités mentales : il faut envisager une étude exhaustive de ces prédicats afin de déterminer si tous peuvent être considérés comme exprimant un genre de PENSER ou un genre de SAVOIR. Goddard (2003), dans un article consacré spécifiquement au primitif anglais THINK, n'explore malheureusement pas les liens de ce primitif avec le primitif SAVOIR [KNOW]. Par contre, il cite Wierzbicka qui remet en cause la distinction vendlerienne bien connue entre deux interprétations du verbe penser : une processuelle et une statique. J'ai montré ailleurs (2007a) que le domaine mental peut être organisé de la même manière que le domaine «matériel », il suffit de repenser les critères de classement en enlevant notamment la primauté du critère « visuel ». Dans l'organisation proposée, la première division est obtenue justement par la différence processuelle état/procès et l'engagement de l'expérienceur dans le procès mental. Savoir est ainsi classé comme un état et penser ${ }^{14}$ comme un procès ce qui va dans le sens de l'hypothèse supra.

Les primitifs FAIRE et ARRIVER catégorisent ce que l'on pourrait appeler le domaine actionnel, tous les deux pouvant être considérés comme deux conceptualisations fondamentales de 'quelque chose se passant dans le monde'. La différence consisterait, là aussi, en une différence de l'agentivité : FAIRE désigne une action effectuée par un agent alors qu'ARRIVER profile un évènement qui n'est pas provoqué par un agent ou dont l'agent n'est pas linguistiquement profilé' avec toutes les variantes possibles de saisie de la chaîne causale ${ }^{15}$.

La structure du domaine volitionnel apparaît comme évidente : elle est organisée autour du primitif VOULOIR et on peut considérer que tous les prédicats volitionnels peuvent être explicités à l'aide de ce primitif.

Enfin, parmi les primitifs regroupés comme des prédicats mentaux, il nous reste encore des primitifs de la perception : ENTENDRE, VOIR et (SE) SENTIR. Le prédicat percevoir est réputé complexe et ne peut donc pas faire partie de la métalangue sémantique naturelle. Il n'en reste pas moins qu'il s'agit d'un catégorisateur pour les primitifs ENTENDRE, VOIR et (SE) SENTIR. Percevoir est une abstraction de ces trois primitifs et cette caractéristique abstraite semble être en conflit avec le statut de primitif. Mais dans la structure sémantique, le fait d'être abstrait de trois primitifs, fait de percevoir un catégorisateur parfait. L'existence du domaine conceptuel de la perception n'est pas discutable ; ce qui peut et doit être discuté, c'est sa structure et ses frontières. Compte tenu du fait que les trois primitifs couvrent les sens humains, on peut considérer qu'ils catégorisent nécessairement le domaine perceptif. Cependant voir, entendre et (se) sentir n'ont pas les mêmes propriétés sémantiques. Voir et entendre, dans leur sens perceptif retenu ici, sont spécifiques (sémantiquement saturés). Je comprends par là que tous les deux concernent des organes sensoriels précis (respectivement les yeux et les oreilles), ils sont non agentifs et possèdent des 
équivalents agentifs regarder et écouter. Rien de tel pour (se) sentir qui ne concerne aucun organe sensoriel précis et qui peut non seulement concerner plusieurs sens mais aussi des valeurs qui relèvent de ce qu'on peut appeler « pré-cognitif», comme par exemple dans sentir venir le danger. (Se) sentir n'a pas non plus d'équivalent agentif. Ce caractère indéfini rend d'ailleurs l'emploi de se sentir dans la MSN difficile et discutable ${ }^{17}$. Pour revenir au problème de la structure du domaine de la perception, on peut dire que tous les prédicats « visuels » peuvent être expliqués à l'aide du primitif VOIR et tous les prédicats « auditifs» peuvent l'être à l'aide du primitif ENTENDRE. Par contre il semble difficile de déterminer quels concepts seraient catégorisés par le primitifs $(\mathrm{SE}) \mathrm{SENTIR}^{18}$, d'où certainement sa polysémie très riche. Par ailleurs, il faudrait vérifier la pertinence des prédicats renvoyant à d'autres sens humains, le goût, l'odorat et le toucher dans la structuration du domaine perceptif pour avoir une organisation complète de ce domaine sémantique.

La place du primitif BOUGER dans la structure sémantique est incertaine. Pour pouvoir la déterminer précisément, il faudrait décider si le domaine du mouvement que ce primitif catégorise fait ou non partie du domaine actionnel abordé supra. La plausibilité de deux possibilités doit encore être déterminée. On peut cependant penser que le domaine conceptuel du mouvement est suffisamment saillant pour être considéré à part. N'oublions pas que l'image linguistique du monde n'est pas nécessairement logique ${ }^{19} \mathrm{et}$ qu'elle semble s'appuyer sur la saillance. Les travaux cognitivistes portant sur l'espace font du domaine de l'espace et du mouvement des domaines fondamentaux structurant d'autres domaines sémantiques, comme par exemple celui du temps. Le mouvement semble être un concept immédiatement accessible que l'on ne pense pas en termes de « faire quelque chose».

Les primitifs regroupés comme 'discours', DIRE et MOT semblent tous deux catégorisateurs. DIRE est conceptualisé en français comme spécifique de l'humain et cette activité est très saillante de la perspective anthropocentrique propre à l'image linguistique du monde. Il serait nécessaire de déterminer ici si l'un de deux primitifs occupe une place supérieure dans la structure sémantique. Dans la logique de l'ordre, DIRE précède MOT, mais cette logique n'est pas nécessairement retenue par l'image linguistique. Wierzbicka (2003) montre que l'on peut définir tous les actes du langage anglais à l'aide du primitif SAY, la même démonstration est possible pour le français. Ici encore, il serait probablement possible de penser l'activité langagière en termes de «faire quelque chose », mais ce n'est pas le cas dans l'image française du monde : le domaine du DIRE est conceptualisé comme indépendant en raison de son importance dans la perspective anthropocentrique.

Les primitifs de l'existence et de la possession IL Y A et AVOIR organisent les domaines fondamentaux qui leurs correspondent.

Enfin, ENDROIT est le seul primitif spatial pouvant être considéré comme catégorisant. Il est indéterminé et les frontières de son application sont relativement floues, mais on pourrait le considérer comme l'organisateur du domaine spatial. L'espace est un concept abstrait et donc non-immédiatement accessible. Il semble que l'on puisse le réduire sémantiquement à ENDROIT. ENDROIT serait catégorisateur de tous les cas d'espace, les autres primitifs spatiaux renverraient à des relations entre les différents endroits et les objets qui les occupent. Si les choses se passent dans l'espace, elles se passent nécessairement dans un endroit. Observons ici aussi, que l'impossibilité d'intégrer un concept comme espace dans la MSN semble être due à son caractère abstrait, celui-ci semblant être corrélé avec une complexité sémantique.

Nous venons de voir les primitifs sémantiques qui jouent un rôle catégorisateur dans la structure sémantique du français (et probablement des autres langues aussi). Contrairement à ce que l'on pourrait s'attendre, il ne s'agit pas d'une grande partie des primitifs et les primitifs catégorisateurs ne sont pas suffisant pour l'organiser l'image linguistique du monde. Ce n'est pas étonnant en soi dans la mesure où les images du monde varient d'une langue à l'autre et ont leurs propres structures sémantiques spécifiques. Seul un petit nombre des structures catégorisantes semble universel. Par ailleurs, nous avons vu que l'on peut retrouver souvent l'opposition actif/passif. Elle est certaine dans le cas de couples FAIRE - ARRIVER, SAVOIR - PENSER, et on peut la supposer également dans les primitifs nominaux QUELQU'UN QUELQUE CHOSE. Il s'agit d'une partition fondamentale qui traverse les domaines sémantiques sans en 
être spécifique. Cependant, cette opposition est à comprendre dans le sens prototypique et scalaire, tel que l'envisage Dowty (op.cit.).

\subsection{Les primitifs spécifiques non catégorisateurs}

Contrairement à des primitifs que nous avons vus jusque là, certains primitifs renvoient à des concepts spécifiques et/ou sémantiquement saturés. Je vais maintenant les passer en revue et montrer leurs spécificités.

Commençons par les primitifs JE et TU. Ils renvoient tous les deux à des humains, ils font donc partie de la catégorie dont le catégorisateur est le primitif QUELQU'UN. Notons que traditionnellement on considère $\mathrm{JE}$ et TU comme des déictiques dépourvus de sens sémantique dont l'interprétation se fait sur le principe instructionnel et contextuel. Dans l'approche en métalangue sémantique naturelle, JE et TU ont un sens qui est indéfinissable, tout comme pour les autres primitifs. Ainsi par exemple le primitif JE sous-tend une interprétation égocentrique du monde ${ }^{20}$ alors que TU présuppose l'autre dans un rapport à JE, dans une relation. On peut ainsi dire que ces deux primitifs catégorisent dans un certain sens, mais il s'agit d'une catégorisation dynamique qui relève des situations et non de la structuration sémantique.

Le primitif GENS, qui renvoie au concept de la pluralité humaine est conceptualisé également comme faisant partie de la catégorie de QUELQU'UN. Contrairement aux primitifs JE et TU, le primitif GENS pourrait, théoriquement, être considéré comme catégorisateur de différentes pluralités humaines, mais cela reste encore à être vérifié. Je précise que ce n'est pas parce que le primitif GENS peut être catégorisé sous le primitif QUELQU'UN, que l'on peut l'expliciter à l'aide de QUELQU'UN. Il renvoie à une idée audelà de la pluralité des personnes humaines, il concerne également une idée de groupe, de généralité et de légitimation. On utilise en effet le primitif gens lorsque l'on a besoin de référer à la doxa : «les gens pensent que c'est bon (ou mauvais)», son sens va donc au-delà de la concaténation de primitifs QUELQU'UN et BEAUCOUP (ou QUELQUES). C'est pour cette raison que l'hypothèse énoncée plus haut sur la possibilité pour le primitif GENS de catégoriser les pluralités humaines, doit encore être vérifiée par une étude systématique des concepts concernés.

Les primitifs VIVRE et MOURIR semblent spécifiques même s'il s'agit de concepts quelque peu vagues. Notons qu'ils ne sont pas sémantiquement saturés de la même manière : VIVRE a des contours sémantiques beaucoup plus flous que MOURIR.

Ce sont les primitifs de l'espace et du temps qui interrogent le plus dans la mesure où ils sont presque tous beaucoup plus spécifiques et saturés que les autres primitifs. Observons tout d'abord les primitifs spatiaux qui sont, en grande partie, relationnels. AU-DESSUS, EN DESSOUS, LOIN, PRES, COTE, DANS et (SE) TOUCHER désignent des relations entre les objets du monde. Il s'agit bien évidemment des relations simples et on peut penser que ces relations renvoient à ce qu'on peut appeler l'expérience immédiate du monde. Il s'agit en effet à chaque fois des concepts nécessaires pour la localisation des objets les uns par rapport aux autres (ou par rapport à un JE qui les perçoit) dans un espace que l'on peut conceptualiser en terme d'ENDROIT, comme nous l'avons vu plus haut. Aucun des primitifs spatiaux en dehors d'ENDROIT, n'est catégorisant. Le primitif déictique ICI peut éventuellement catégoriser situationnellement des ENDROITs, de la même manière que les primitifs JE et TU catégorisent situationnellement des PERSONNEs. Le concept du temps lui-même n'est pas primitif, il s'agit encore une fois d'une abstraction. Il est difficile de ramener le temps au concept primitif de MOMENT ou de FOIS, et aucun des primitifs du domaine temporel ne semble catégorisant. On observe donc une dissymétrie avec le domaine spatial qui a le catégorisateur ENDROIT. Le primitif déictique MAINTENANT s'interprète de la même manière que le primitif déictique spatial ICI. Les primitifs temporels spécifiques sont, dans un certain sens, des localisateurs, tout comme des primitifs spatiaux, ils permettent de localiser les objets (ou les évènements) dans le temps. L'existence du domaine temporel n'est pas discutable mais il faut encore investiguer sa structure interne. Il semble nécessaire de considérer le prédicat temps comme catégorisateur en français, même si cela nous obligerait à penser que la structuration entière du domaine temporel est culturellement spécifique. 
En dehors de ces primitifs que nous venons de voir et dont la saturation sémantique ne permet pas de jouer le rôle catégorisateur, la place de certains autres est discutable, mais nous les verrons plus tard.

\subsection{Les primitifs « modalisateurs »}

La troisième catégorie de primitifs qui se dégage concerne les primitifs qui semblent pas catégoriser et qui ne sont pas spécifiques non plus. Ces primitifs semblent avoir un rôle transverse de modalisation des concepts de tous les domaines. On comprendra ici par modalisation, des modifications des concepts auxquels les modalisateurs s'appliquent, peut importe la nature de cette modalisation. Les modifications sont de différentes sortes et cette catégorie de primitifs est probablement la plus difficile à explorer.

Commençons par le primitif déterminant $\mathrm{CE}$. Il renvoie à une idée de monstration, il peut en cela être considéré comme déictique au même titre que la triade énonciative présente dans la MSN : JE, ICI et MAINTENANT. Cependant, il est différent. Contrairement aux autres primitifs déictiques, il n'est pas autonome, il s'applique aux primitifs nominaux ${ }^{21}$ en les modifiant par l'idée de la monstration. On peut expliquer cette modalisation ainsi : dans la formulation « cette chose », " chose » est différente de toutes les autres choses, parce qu'elle est indiquée, soustraite de l'ensemble des choses.

De la même manière les quantificateurs peuvent modifier tous les primitifs nominaux ${ }^{22}$. On pourrait s'interroger sur l'existence d'un domaine sémantique de quantité. Cela dit, la mise en évidence très probable d'un tel domaine ne changerait pas le fait que celui-ci serait de toute manière modificateur.

Le concept de la vérité, représenté dans la MSN française par le primitif VRAI fonctionne comme un modalisateur. Il ne catégorise pas mais qualifie les différents autres concepts, notamment ceux de domaines de PENSER et de DIRE. Notons par ailleurs que la présence de ce primitif dans la MSN est discutable et devrait être éclairci, notamment à cause de l'interprétation très variée de ce concept dans les différentes cultures.

Le place des évaluateurs BIEN et MAL dans la structuration sémantique peut être abordée de deux manières différentes. On pourrait partir du principe qu'il existe une moralité «primaire» qui sous-entend un domaine conceptuel «moral», mais cette solution semble être difficile à justifier. Krzeszowski (1997) montre que tous les objets de l'image linguistique du monde sont chargés axiologiquement. Par conséquent il serait peut-être alors plus judicieux de considérer les évaluateurs BIEN et MAL comme des modalisateurs qui modifient les autres objets au niveau de l'orientation axiologique. Les évaluateurs BIEN et MAL peuvent s'appliquer à tous les éléments nominaux et certains éléments verbaux.

De la même manière, les descripteurs GRAND et PETIT ne sont pas catégorisateurs mais ils modifient les concepts nominaux.

Les primitifs TRES, PLUS et la négation NE...PAS ne peuvent pas être envisagés autrement que comme des modalisateurs, ils modifient en effet les autres éléments conceptuels.

Enfin, deux autres primitifs MEME et AUTRE, sont assez problématiques. MEME renvoie nécessairement à une idée d'identité et AUTRE à une idée d'altérité. Ces deux primitifs sont employés dans les explicitations verbales dans le sens plutôt relationnel : « même que » et « autre que » servent donc à modifier.

Le primitif PEUT-ETRE, tout comme le concept logique SI sont également des modalisateurs, mais ils se distinguent des autres primitifs modalisateurs par le fait qu'ils ne modalisent pas des concepts particuliers, mais des clauses ${ }^{23}$.

Les primitifs modalisateurs soulèvent une interrogation plus générale au sujet de la relation des primitifs sémantiques et de la structure sémantique elle-même. Une des propriétés fondamentales des primitifs sémantiques est leur caractère immédiatement accessible. Les notions comme la probabilité ou la quantité sont des notions qui procèdent d'une activité d'abstraction, elles sont donc complexes. Même si l'on peut postuler, théoriquement, l'existence de tels domaines, il n'est pas certain qu'ils existent dans l'image linguistique du monde, il est possible par contre, conformément à l'hypothèse exposée ici, que les concepts comme peut-être ou encore autre, traversent les autres domaines, sans constituer eux-mêmes, 
des domaines spécifiques. Nous serions alors devant deux possibilités d'approche différentes : d'une part, les constructions théoriques suivant une approche «scientifique», d'autre part, l'approche naturelle ou «naïve», qui cherche à découvrir ${ }^{24}$ les structures présentes, suivant la logique naturelle parfois contradictoire.

\subsection{Les primitifs à déterminer}

La place et la fonction de certains primitifs dans la structuration sémantique n'est pas claire et nécessite d'être étudiée davantage.

Le primitif CORPS est un des primitifs les plus récents et ce qu'on retrouve dans les publications sur la MSN n'est pas suffisant pour établir la place de ce primitif dans la structuration de l'image du monde. Il n'est même pas clair si ce primitif concerne uniquement un corps humain ou bien les corps des autres êtres. Il est nécessaire d'étudier les rapports de ce primitif avec les primitifs QUELQU'UN et CHOSE. Ces rapports ne sont pas simples dans la mesure où CORPS peut renvoyer aussi bien à QUELQU'UN qu'à CHOSE (cf. Wierzbicka, 1976).

Le statut du primitif POUVOIR n'est pas très clair non plus. L'indéfinissabilité des primitifs pose une fois de plus un problème. Le verbe pouvoir en français est polysémique et les énoncés-exemples ne lèvent pas l'ambiguité, alors que l'on peut devoir supposer qu'une seule valeur est retenue. Ainsi par exemple, dans l'énoncé MSN emprunté à Peeters \& alii (2006:180) :

\section{Si je pouvais le faire, je le ferai.}

deux lectures sont possibles : on peut interpréter le primitif en terme de capacité (je suis en capacité de le faire) et en terme d' «autorisation» (quelque chose d'extérieur le permet). Les deux valeurs sont spontanément accessibles. La difficulté de choix de valeur demande un examen approfondi de ce primitif, même si son rôle modalisateur semble évident dans la mesure où, dans les explicitations verbales, le primitif POUVOIR est toujours suivi par un autre verbe. C'est d'ailleurs pour cette raison certainement que Wierzbicka (1996) parle de « métaprédicat».

Le primitif PARCE QUE semble être spécifique, non catégorisateur et non modalisateur. Son rôle est de mettre en rapport des énoncés et probablement de modaliser un énoncé par rapport à l'autre. Cependant ce point nécessite encore d'être analysé.

Enfin, dans l'état actuel de la recherche, il est difficile de déterminer le rôle et la place des primitifs PARTIE DE et GENRE DE.

\section{Penser les primitifs dans la structure sémantique de l'image linguistique du monde}

Dans la plupart des publications qui leur sont consacrées, les primitifs sémantiques sont étudiés dans le cadre de la métalangue sémantique naturelle et de ses applications explicitantes. Pourtant on a souligné à maintes reprises que les primitifs appartiennent à la langue ${ }^{25}$ contrairement aux éléments des métalangages abstraits que le mentalais de Fodor (1975) ou encore le métalangage abstrait de Jackendoff (op.cit.). Cette filiation impose une interrogation nécessaire sur la place des primitifs sémantiques dans les langues dont ils sont extraits.

J'ai essayé ici de montrer un premier aperçu de la place des primitifs sémantiques dans la structure sémantique du français. Comme cela a été annoncé, il s'agit d'une approche du problème, d'un projet de recherches systématisées. Nous pouvons d'emblée voir que le statut des primitifs et le rôle qu'ils jouent dans la structure sémantique ne sont pas unifiés. Trois cas de figure se présentent: les primitifs catégorisateurs qui structurent des domaines et donc organisent d'autres concepts, les primitifs spécifiques dont le statut dans les domaines correspondants est égal au statut des unités non primitives et enfin les primitifs modalisateurs qui modifient les autres unités conceptuelles, qu'elles soient primitives ou non. Il est peut-être trop tôt pour en tirer des conclusions au sujet des primitifs mais, il me semble, que 
cette disparité va dans le sens de réserves qu'U. Eco (1997) adresse à la théorie des primitifs. Pour Eco, les primitifs renverraient à des expériences primordiales, toutefois le fait que les primitifs renvoient à ce type d'expérience ne signifie pas que les concepts de ces expériences ne soient pas interprétables. Cette réserve ne remet pas en cause les postulats de la métalangue sémantique naturelle, les primitifs étant indéfinissables selon les règles définitoires de la MSN, mais elle nous mène à une approche d'interprétation nécessaire des primitifs en dehors des règles de la MSN. Il faut ouvrir les recherches sur les primitifs en tant qu'éléments de la langue. Les publications portant sur la MSN (p.ex. Goddard, 2003), mettent souvent en évidence le caractère circulatoire et obscur des définitions lexicographiques des primitifs. Pour cette raison, il est nécessaire de faire la différence terminologique entre la définition et l'interprétation proposée par Eco. Les primitifs doivent être approchés. Leur analyse dans la langue devrait nous permettre de comprendre les problèmes créés par certaines modifications de la MSN française. Ainsi par exemple le changement récent de la catégorie des primitifs évaluateurs BON et MAUVAIS en BIEN et MAL (Peeters, 2006) devrait mener nécessairement à une interrogation portant sur la valeur précise qui est sélectionnée, les adjectifs et les adverbes semblent en effet sélectionner des valeurs différentes dans la langue. Il apparaît également comme nécessaire, d'instaurer une perspective francophone des études sur la métalangue sémantique naturelle. En effet, certains primitifs ne semblent poser aucun problème en anglais, alors qu'ils sont problématiques en français et/ou dans d'autres langues. C'est le cas, par exemple du primitif FEEL qui est problématique en français, en polonais, en luxembourgeois, en chinois et probablement dans d'autres langues. Le changement des évaluateurs signalé plus haut n'apporte pas d'interrogation en anglais où la forme adjectivale se confond avec la forme adverbiale. Les interrogations concernant le français devrait aider à interpréter les primitifs évaluateurs.

Nous avons remarqué que les primitifs sémantiques semblent être incompatibles avec le caractère abstrait des unités linguistiques. Ainsi par exemple espace ne peut pas être primitif, parce qu'il doit être pensé, alors qu'ENDROIT semble être directement accessible. Cette incompatibilité avec l'abstraction concorde avec la vision d'Eco des primitifs, en tant que conceptualisateurs des expériences primordiales. La particularité des primitifs dans la structure sémantique consisterait en le fait qu'ils constituent un minimum conceptuel indispensable pour organiser et dire le monde, un ensemble minimal immédiatement accessible. En tant que tels, les primitifs sémantiques n'ont besoin ni d'avoir le même statut dans la structure sémantique, ni de jouer un même rôle. L'incompatibilité entre le caractère abstrait et les primitifs devrait être analysée de manière approfondie, notamment par rapport à la saturation sémantique. L'analyse de cette incompatibilité est nécessaire d'autant plus qu'elle semble paradoxale si l'on comprend l'abstraction comme un phénomène de subduction de la théorie guillaumienne (Picoche, 1986), la subduction se traduisant par un appauvrissement sémantique.

Un certain nombre de domaines conceptuels (ou sémantiques) a été postulé dans cette présentation. L'existence sémantique de quelques-uns de ces domaines apparaît comme évidente, comme par exemple celle du domaine cognitif, volitionnel, actionnel, celle de quelques autres reste encore à être démontrée. Pour les domaines catégorisés par les primitifs sémantiques, il faudra établir et tester un modèle d'explicitations verbales qui permettra de décrire, de la même manière, tous les éléments du domaine en question, conformément au postulat de Wierzbicka (1985) sur l'iconicité de la définition par rapport au domaine conceptuel. Il semble d'emblée évident qu'un tel procédé risque d'être difficile pour les domaines très étendus, comme par exemple celui de CHOSE. Les difficultés rencontrées aideront nécessairement à mettre en évidence une structure plus détaillée du domaine.

Enfin, nous avons également abordé le problème de différents niveaux de catégorisation : universel et culturellement spécifique. Les primitifs, par leur nature-même, imposent une catégorisation universelle. Sur la base de celle-ci se construisent les structurations culturellement spécifiques qu'il faudra mettre en évidence pour compléter l'image linguistique du monde contenue en français. 


\section{Références bibliographiques}

Bogusławski, A. (1991). Semantic primes for agentive relations. Lingua Posnaniensis, XXXII-XXXIII, 39-64.

Bossong, G. (1998). Le marquage différentiel de l'objet dans les langues d'Europe. In Feuillet, J. (éd). Actance et valence dans les langues de l'Europe, Berlin/New York : Mouton de Gruyter, 193-258.

Dąbrowska, E. (1994). Nominative and dative experiencers: Two folk theories of the mind. Linguistics, 32/6, 10291054.

Dowty, D. (1991). Thematic Proto-Roles and Argument Selection. Language, 67, 547-619.

Durst, U. (2003). The Natural Semantic Metalanguage approach to linguistic meaning. Theoretical Linguistics, 29, 157-200.

Eco, U. (1997). Kant et ornithorynque. Paris : Grasset.

Fodor, J.A.(1975). The language of thought. Cambridge: Harvard University Press.

Goddard, C. (1994). Semantic Theory and Semantic Universals. In Goddard, C., Wierzbicka, A., Semantic and lexical universals. Theory and empirical findings. Amsterdam/Philadelphia: John Benjamins, 7-29.

Goddard, C. (1997). The universal syntax of semantic primitives. Language Sciences, 19/3, 197-207.

Goddard, C. (2002). The search for the Shared Semantic Core of All Languages. In Goddard, C., Wierzbicka, A., Meaning and Universal Grammar. Theory and empirical findings. Vol. I. Amsterdam/Philadelphia : John Benjamins. 5-40.

Goddard, C. (2003) Thinking across languages and cultures: Six dimension of variation. Cognitive Linguistics, 14 , $2 / 3,109-140$.

Goddard, C., Wierzbicka, A. (1994), Semantic and lexical universals. Theory and empirical findings. Amsterdam/Philadelphia: John Benjamins, 7-29.

Goddard, C., Wierzbicka A. (2002). Meaning and Universal Grammar. Theory and empirical findings. Vol. I. Amsterdam/Philadelphia : John Benjamins, 41-85.

Jackendoff, R. (1985). Semantics and Cognition. Cambridge : The Mit Press.

Koselak, A. (2003). La sémantique naturelle d'Anna Wierzbicka et les enjeux interculturels. Questions de Communication, 4, 83-97.

Koselak, A. (2007a), Sémantique des sentiments «quand je pense à toi, je ressens quelque chose de mauvais 》 en français et en polonais, Thèse de Doctorat, Université Paul Verlaine de Metz, dir. C. Masseron, A. Petitjean.

Koselak, A. (2007b), Sources et tradition polonaise en sémantique cognitive. CORELA, Numéros spéciaux, Cognition, discours, contextes. Accessible en ligne à l'URL : http://edel.univpoitiers.fr/corela/document.php?id=1626.

Koselak, A. (2008), Un modèle descriptif des affects. L'exemple de la jalousie et de zazdrość. In Bogacki, K., Cholewa, J., Rozumko, A., Methods of lexical analysis : theoretical assumption and practical applications. Wydawnictwo Uniwersyteckie: Białystok. 241-249.

Krzeszowski, T. (1997). Angels and devils in hell. Warszawa : Energeia.

Langacker, R. (1987). Foundations of cognitive grammar. Vol. I. Theoretical Prerequisites. Stanford: Stanford University Press.

Maćkiewicz, J. (1999). Co to jest językowy obraz świata? Etnolingwistyka, 11, 7-24.

Peeters, B. (2002). La "métalangue sémantique naturelle» au service de l'étude du transculturel. Travaux de linguistique 45, Gand, 83-101.

Peeters, B. (ed). (2006). Semantic Primes and Universal Grammar. Empirical evidence from the Romance Languages. Amsterdam/Philadelphia : John Benjamins.

Picoche, J. (1986). Structures sémantiques du lexique français. Paris : Nathan. 
Sériot, P. (2005). Oxymore ou malentendu? Le relativisme universaliste de la métalangue sémantique naturelle d'Anna Wierzbicka. Cahiers Ferdinand de Saussure 58, 23-43.

Wierzbicka, A. (1972). Semantic Primitives. Frankfurt/Main: Athenäum.

Wierzbicka, A. (1976). Mind and body. In MacCawley, J.D. (ed). Notes from the Linguistic Underground. Syntax and Semantics 7. New York: Academic Press, 129-157.

Wierzbicka, A. (1988). The semantics of grammar. Amsterdam/Philadelphia: John Benjamins.

Wierzbicka, A. (1989). Semantic Primitives and Lexical Universals. Quaderni di Semantica, X/1, 103-121.

Wierzbicka, A. (1985). Lexicography and conceptual analysis. Tucson : Karoma.

Wierzbicka, A. (1996). Semantics. Primes and universals. Oxford/New York : Oxford University Press.

Wierzbicka, A. (2003). Cross-Cultural Pragmatics. Berlin/New York: Mouton de Gruyter.

\footnotetext{
${ }^{1}$ Je comprends par là qu'ils sont très peu définis et qu'ils peuvent par conséquent renvoyer à un nombre important de concepts.

${ }^{2}$ L'usage veut que l'on écrive les primitifs sémantiques avec de petites majuscules.

${ }^{3}$ Voir, en français, par exemple Peeters (2002), Koselak (2003, 2007b). Les principales publications anglophones : Wierzbicka (1996), Goddard \& Wierzbicka (1994, 1002), Peeters (2006). On peut trouver également une bibliographie exhaustive à l'adresse suivante : http://www.une.edu.au/bcss/linguistics/nsm/
}

${ }^{4}$ Voir, par exemple, Langacker (1987).

${ }^{5}$ Il peut s'agir aussi bien des lexèmes (le cas le plus fréquent) que des unités plus petites (morphèmes) ou plus grandes (phrasèmes).

${ }^{6}$ Voir Goddard (2002) pour le récapulatif des langues étudiées du point de vue de la MSN.

${ }^{7}$ Voir, par exemple Goddard (1997).

${ }^{8}$ Voir Koselak (2008) pour l'exemple du modèle descriptif des affects.

${ }^{9}$ Voir Durst (2003) pour l'historique du développement de la MSN.

${ }^{10}$ Ma traduction.

${ }^{11}$ Même si parfois ils peuvent être réduits à l'état de chose, dans ce cas le verbe réduire indique le changement de statut.

${ }^{12}$ On pourrait discuter le label de cette catégorie dans la mesure où il est difficile de penser que, par exemple, (se) sentir serait un prédicat mental.

${ }^{13}$ Les notions d'agentivité et de patientivité sont ici à prendre au sens que leur donne Dowty (1991).

${ }^{14} \mathrm{~J}$ 'ai distingué alors deux valeurs habituellement mis en évidence : penser que et penser à. Il me semble maintenant que les raisons qui m'ont conduit à cette distinction ne sont pas valables.

${ }^{15}$ Voir Wierzbicka (1988) pour des exemples des saisies originales en russe.

${ }^{16}$ Voir également Bogusławski (1991).

${ }^{17}$ Voir Koselak, 2007a.

${ }^{18}$ Comme il est difficile de déterminer quelle valeur de (se) sentir est mobilisée dans la MSN.

${ }^{19} \mathrm{Au}$ sens scientifique de ce terme. L'image du monde suit sa propre logique, et parfois plusieurs logiques contradictoires.

${ }^{20}$ La notion d'égocentrisme, utilisée ici, n'a aucune connotation psychologique, mais uniquement anthropologique. L'homme interprète le monde, par rapport à lui-même, d'où par exemple les postulats sur les bases corporelles de la 
métaphorisation ou l'idée que l'image linguistique du monde nous apprend plus sur celui qui la crée (l'homme) que sur le monde lui-même.

${ }^{21}$ Dans certaines explicitations verbales on retrouve l'allolèxe CELA syntaxiquement autonome (donc remplissant la fonction nominale) mais servant d'ana- ou de cataphore.

${ }^{22}$ Goddard \& Wierzbicka (2002) parlent de specifiers.

${ }^{23}$ J'utilise ici le terme clause au sens d'énoncé simple.

${ }^{24}$ L'idée de « découverte » en tant que démarche scientifique, semble inacceptable pour certains auteurs. Par exemple Sériot (2005) semble refuser la possibilité de découverte aux sciences humaines alors que les démarches de découverte ne posent pas de problème dans les sciences dites « exactes ».

${ }^{25} \mathrm{Et}$ au langage également. C'est d'ailleurs là que réside l'originalité des primitifs sémantiques qui les distinguent des sèmes ou noèmes, tels que ces concepts ont été conçus dans les sémantiques compositionnels ( $c f$. Koselak, 2007b). 